\title{
Characteristics of attempted suicide patients admitted in Jigme Dorji Wangchuck National Referral Hospital from January 2014 to September 2018
}

\author{
Zimba Letho', Neyzang Wangmo², Kuenzang ${ }^{3}$ \\ ${ }^{1}$ Medical Education and Research Unit, Jigme Dorji Wangchuck National Referral Hospital, Thimphu, Bhutan \\ ${ }^{2}$ Medical Education Center for Research, Innovation and Training, Khesar Gyalpo University of Medical Sciences of Bhutan, Thimphu, Bhutan \\ ${ }^{3}$ Faculty of Nursing and Public Health, Khesar Gyalpo University of Medical Sciences of Bhutan, Thimphu, Bhutan
}

\begin{abstract}
Introduction: Attempted suicide is a major public health problem with a prevalence rate of approximately $2.7 \%$ worldwide. There is an increasing trend of attempted suicide in Bhutan. It is a strong clinical predictor of eventual suicide deaths and results in significant use of health care resources. This study aimed to describe the characteristics of attempted suicide patients admitted in Jigme Dorji Wangchuck National Referral Hospital from January 2014 to September 2018. Methods: This descriptive record review analyzed 72 clinical records of attempted suicide patients. The characteristics of attempted suicides are described using detailed illness history of patients who were admitted in the psychiatric and medical wards. Results: The result showed a higher rate of attempted suicide in females $(56.9 \%)$ and highest in the age range between 21 and 30 years. The percentage of patients from the western region of the country was higher (48.6\%). The median duration of hospital stay was 5 days (range 1-74). There was a slightly higher number of attempted suicide admissions in the cold season. Pre-existing mental health disorders, mostly depressive disorder, was the major underlying cause of attempted suicide (55.5\%). The predominant method used for attempted suicide was self-poisoning on medication (76.3\%) with the common medicine being Paracetamol. Conclusions: To prevent attempted suicide and eventual suicide, there is a need to strengthen early detection of depressive illnesses and regulate the accessibility of over-the-counter drugs like Paracetamol.
\end{abstract}

Keywords: Attempted suicide; Bhutan; Suicide.

\section{INTRODUCTION}

Attempted suicide is a nonfatal, self-directed, potentially injurious behavior with an intent to die as a result of the behavior even if the behavior does not result in injury ${ }^{1}$. Suicide is one of the major public health issues in the world. About one million people die from suicide annually, accounting for $1.4 \%$ of all deaths in the world ${ }^{2}$. The global lifetime prevalence rate of attempted suicide is approximately $2.7 \% \%^{3}$. According to the annual report of the World Health Organization (2014), the highest number of suicide cases was in the South East Asia Region and Bhutan was ranked to have the fifth-highest suicide rate in the Asia-Pacific Region ${ }^{2}$. In Bhutan, as per the 2018 Annual Health Bulletin (AHB) of the Ministry of Health, suicide deaths were found to be among the top six leading cause of deaths after alcoholic liver disease, circulatory diseases, cancers, respiratory diseases, and road accident deaths. Moreover, in 2017, Thimphu had 24 cases of attempted suicide,

Corresponding author:

Zimba Letho

zletho@jdwnrh.gov.bt which was highest in the country ${ }^{4}$. In addition, the record with Royal Bhutan Police from 2009 to 2013 showed that there were 361 suicidal deaths and the attempted suicide cases ranged from 502 to 682 with the ratio of approximately 2:1 (attempted suicide : suicide) $)^{5}$. This is in line with a study conducted on suicide trends in Bhutan, which showed an increasing trend of suicidal rate in Bhutan $^{6}$.

Given the increasing trend of suicide and attempted suicide cases, the suicide prevention action plan identified the key risk factors of attempted suicide as mental problems (84\%), stressful events $(68 \%)$ and substance abuse $(59 \%)$ followed by domestic violence $(46 \%)^{5}$. The Gross National Happiness (GNH) survey conducted in 2010 showed higher cases of attempted suicide in urban societies ${ }^{7}$. Moreover, evidence shows that the strong clinical predictor of eventual suicide deaths was found to be previous attempted suicide which also results in negative consequences such as injury, hospitalization, loss of freedom and causes huge financial burden ${ }^{1-3}$.

It is found that about one-third of people who attempt suicide will repeat the attempt in a year, and up to $10-15 \%$ of those who attempt suicide eventually die by suicide ${ }^{8}$. However, due to the lack of information on characteristics of attempted suicide, 
it is important to generate baseline knowledge on attempted suicide in Bhutan. Therefore, this study was aimed to describe the characteristics of attempted suicide patients admitted in Jigme Dorji Wangchuck National Referral Hospital (JDWNRH) from January 2014 to September 2018.

\section{METHODS}

Descriptive record review was used to describe the characteristics of attempted suicide patients admitted in JDWNRH from January 2014 to September 2018. JDWNRH is a tertiary level hospital and the only hospital in the country that caters specialized psychiatric services.

All patients with attempted suicide were admitted in psychiatric and medical wards. Patients' baseline information was collected from the indoor registers of the respective wards. In addition, details of the patient's illness history were extracted from the files shelved at the medical record unit of JDWNRH.

Data was collected after obtaining administrative clearance from Ministry of Health and ethical clearance from the Research Ethics Board of Health of Bhutan (REBH/ Approval/2018/009). Data was collected using a question guide to capture the necessary informationfor the study.

Data was coded, double entered and validated in Epi data version 3.1. Then it was analyzed using Stata version 13.1. Descriptive statistics was used to present characteristics of attempted suicide admitted in JDWNRH which is described in terms of frequency, percentage, median, inter quartile range and range.

\section{RESULTS}

As shown in Table 1, a total of 72 patients were admitted with the diagnosis of attempted suicide in JDWNRH, of which $56.9 \%$ were females and highest was in the age range between 20 and 30 years with a median age of 24.5 (range 12-69). The minimum and maximum age of the patients was 12 and 69 years respectively. With regard to the geographic distribution/region, $48.6 \%$ were from the western region followed by the eastern and central regions. The median duration of hospital stay of attempted suicide patients was 5 days (range 1-74). There was not a remarkable seasonal variation of attempted suicide. However, relatively higher attempted suicide admission was found in spring $(29.2 \%)$ and winter (26.4\%).

As shown in Table 2, the pre-existing psychiatric disorders accounted for $55.5 \%$ of the cause of attempted suicide. Among psychiatric disorders, major depressive disorder was the most common disorder in patients with attempted suicide $(27.8 \%)$, followed by anxiety disorder (11.1\%) and substance use disorders $(6.9 \%)$. Other underlying causes were found to be stressful life events $(19.5 \%)$, relationship issues $(13.9 \%)$ and other medical conditions $(11.1 \%)$.

The frequency and percentage of methods used for attempted suicide are summarized in Table 3. Self-poisoning
Table 1. Demographics, duration of hospital stay and seasonal variation of attempted suicide patients admitted in JDWNRH from January 2014 - September $2018(n=72)$

\begin{tabular}{|c|c|c|}
\hline Characteristics & $n$ & $\%$ \\
\hline \multicolumn{3}{|l|}{ Gender } \\
\hline Male & 31 & 43.1 \\
\hline Female & 41 & 56.9 \\
\hline \multicolumn{3}{|c|}{ Age(Median = 24.5, Range 12-69) } \\
\hline$\leq 20$ years & 21 & 29.2 \\
\hline $20-30$ years & 30 & 41.7 \\
\hline$\geq 30$ years & 21 & 29.1 \\
\hline \multicolumn{3}{|l|}{ Region } \\
\hline Eastern & 20 & 27.8 \\
\hline Western & 35 & 48.6 \\
\hline Central & 17 & 23.6 \\
\hline \multicolumn{3}{|c|}{ Duration of hospital stay $($ Median $=5$, Range 1-74) } \\
\hline$\leq 4$ days & 35 & 48.6 \\
\hline 5 to 10 days & 27 & 37.5 \\
\hline$\geq 10$ days & 10 & 13.9 \\
\hline \multicolumn{3}{|l|}{ Seasonal variation } \\
\hline Winter & 19 & 26.4 \\
\hline Spring & 21 & 29.2 \\
\hline Summer & 15 & 20.8 \\
\hline Autumn & 17 & 23.6 \\
\hline
\end{tabular}

was seen in $76.3 \%$ of patients of which $31.9 \%$ had taken paracetamol, $15.3 \%$ had taken amitriptyline, $11.1 \%$ had taken other psychotropic drugs, $6.9 \%$ had taken pesticide and $11.1 \%$ had taken others substances like aconite, traditional medicines, steroids and warfarin. Other methods used were strangulation (15.3\%), jumping in the river (5.6\%) and cutting the wrist $(2.8 \%)$.

Table 2. Underlying causes of attempted suicide in patients admitted in JDWNRH from January 2014 - September $2018(n=72)$

\begin{tabular}{lll} 
Underlying Causes & $\boldsymbol{n}$ & \% \\
\hline Pre-existing Psychiatric Disorders & & \\
Major Depressive Disorder & 20 & $27.8 \%$ \\
Anxiety Disorder & 8 & $11.1 \%$ \\
Substance Use Disorder & 5 & $6.9 \%$ \\
$\quad$ Others* & 7 & $9.7 \%$ \\
Stressful Life Events & 14 & $19.5 \%$ \\
Relationship Issues & 10 & $13.9 \%$ \\
Other Medical Conditions ${ }^{\dagger}$ & 8 & $11.1 \%$ \\
\hline
\end{tabular}

"Schizophrenia, bipolar affective disorder, seizure disorders, personality disorder and gender dysphoria.

${ }^{\dagger}$ Tuberculosis, brain tumor, heart diseases, chronic kidney disease and HIV/AIDS. 
Table 3. Methods used amongst attempted suicide patients admitted in JDWNRH from January 2014 - September $2018(n=72)$

\begin{tabular}{lll}
\hline Methods & $\boldsymbol{n}$ & $\mathbf{\%}$ \\
\hline Self-poisoning & & \\
$\quad$ Paracetamol poisoning & 23 & $31.9 \%$ \\
$\quad$ Amitriptyline poisoning & 11 & $15.3 \%$ \\
$\quad$ Other psychotropic drug poisoning* & 8 & $11.1 \%$ \\
$\quad$ Pesticide poisoning $^{\text {Others }}{ }^{*}$ & 5 & $6.9 \%$ \\
Strangulation & 8 & $11.1 \%$ \\
Jumping in the river & 11 & $15.3 \%$ \\
Cutting the wrist & 4 & $5.6 \%$ \\
\hline
\end{tabular}

*Lorazepam, olanzapine and opioids.

*Aconite, traditional medicine, steroids, warfarin and unknown medicines.

\section{DISCUSSION}

The aim of this study was to describe the characteristics of attempted suicide patients admitted in JDWNRH from January 2014 to September 2018. In line with the global trend ${ }^{2}$ and prior findings ${ }^{9,10}$, frequency of attempted suicide was higher in females and in the younger population (age group of 20 to 30 years). The frequency of attempted suicide is higher in females because they have higher rates of depressive disorder as compared to males ${ }^{11}$ and depressive disorder is found to be main underlying cause of attempted suicide in this study.

It was observed that the number of attempted suicides in spring and winter was relatively higher than in summer and autumn. This is in line with studies conducted in Europe and Middle East Asia where higher rates of attempted suicide was noted in spring and winter ${ }^{10-11}$. The reason for such seasonal variation is likely due to the biological, cultural and bio-climatic factors ${ }^{12}$.

Over half of the patients who attempted suicide had pre-existing psychiatric disorders contributing to their suicide attempt, followed by stressful life events, relationship issues and other medical conditions. This finding is in line with a study conducted in India which showed that four broad psychiatric disorders that put people at risk for attempted suicide are mood disorders, substance abuse disorders, schizophrenia and anxiety disorders ${ }^{11}$. In this study, the pre-existing psychiatric disorders that lead to attempted suicide were major depressive disorder, anxiety disorder, substance use disorder and other disorders like schizophrenia, seizure disorders, personality disorder and gender dysphoria. Recent stressful events and relationship issues that accounted for attempted suicide in this study includes recent bereavement, separation or divorce, early loss and reduced social support. The prevalence of medical conditions varies from $25 \%$ to $70 \%$ among those who attempt suicide and it appears to be an important factor in attempted suicide ${ }^{13}$. There is a large number of literature that supports the notion that the presence of a medical condition is a risk factor for suicidal behavior ${ }^{14}$. In this study, $11.1 \%$ of those who attempted suicide had a medical condition. Medical conditions most often associated with attempted suicide include cancer, epilepsy, chronic kidney diseases, musculoskeletal disorders and HIV/AIDS ${ }^{13-14}$.

Self poisoning was the predominant method used for attempted suicide, accounting for $76.3 \%$ of all methods. This finding is comparable to previous study results reported in Asia $(69-86 \%)^{11-12}$. The most commonly used substance for self poisoning was paracetamol, which is similar to previous studies where its use is predominantly seen in adolescents and young adults ${ }^{15-16}$. In JDWNRH, amitriptyline is a commonly prescribed drug for those diagnosed with depressive disorder and the medication refill is usually given for one month. Since major depressive disorder is a common underlying cause of attempted suicide and due to poor domiciliary care in terms of medication supervision, $15.3 \%$ of amitriptyline self poisoning was noted in this study. The use of various medicines for self poisoning as a method to attempt suicide probably reflects the easy accessibility and availability of paracetamol in pharmacy outlets and the prescribing practices in the hospital.

\section{LIMITATIONS}

This study has several limitations. Firstly, the descriptive record review design does not provide causal relationship. Secondly, the data was collected from a single hospital, thereby limiting the generalizability of the finding to other hospitals. Thirdly, we obtained data from the patients who were admitted in the hospital, missing the patients who attempted suicide and presented in emergency department and other out patients departments.

\section{CONCLUSIONS}

This study is the first of its kind in Bhutan and provides knowledge about characteristics of patients who attempt suicide. Early screening and treatment of psychiatric disorder, particularly major depressive disorder, needs to be strengthened to prevent attempted suicide and eventual suicide. Similarly, regulations for the access to self poisoning medicines need to be improved.

\section{ACKNOWLEDGEMENTS}

The authors would like to thank the Department of Psychiatry and Medical Record Unit of JDWNRH for allowing access to clinical records of patients for data collection. We would also like to thank Mr. Sonam Tobjur, staff nurse of psychiatric ward, Mr. Dendup, clinical counseling student, and four other GNM students (graduated in 2019) for rendering help during data collection. We extend our heartfelt appreciation to all the mentors and participants of Bhutan-Structured and Mentoring Approach to Research Training (B-SMART) 2018. 


\section{REFERENCES}

1. Ballesteros MF, Webb K, McClure RJ. A review of CDC's Web-based Injury Statistics Query and Reporting System (WISQARS ${ }^{\mathrm{TM}}$ ): Planning for the future of injury surveillance. J Safety Res. 2017; 61:211-5. [Full Text | DOI]

2. McManus S, Gunnell D. Trends in mental health, nonsuicidal self-harm and suicide attempts in 16-24-year-old students and non-students in England, 2000-2014. Soc Psychiatry Psychiatr Epidemiol. 2020; 55(1):125-8. [Full Text | DOI]

3. Nock MK, Borges G, Bromet EJ, Alonso J, Angermeyer M, Beautrais A, et al. Cross-National prevalence and risk factors for suicidal ideation, plans and attempts. Br J Psychiatry. 2008; 192: 98-105. [Full Text | DOI]

4. Ministry of Health. Annual Health Bulletin, 2018. Thimphu, Bhutan: Policy \&Planning Division, Ministry of Health; 2018.21p. [Full Text]

5. Dorji G, Choki S, Jamphel K, Wangdi Y, Chogyel T, Dorji $\mathrm{C}$, et al. Policy and governance to address depression and suicide in Bhutan: the national suicide-prevention strategy. WHO South-East Asia J Public Health. 2017; 6(1):39-44.

6. Lhadon K. Suicide trends in Bhutan from 2009 to 2013. Journal of Bhutan Studies. 2014; 30. [Full Text]

7. Brassard C. Disaster Governance, Inequality and Poverty Alleviation in Bhutan: Towards Integrated and Preventive Policies. In Development Challenges in Bhutan. Springer, Cham. 2017; pp. 171-201. [Full Text]

8. Mościcki EK. Epidemiology of completed and attempted suicide: toward a framework for prevention. Clin Neurosci Res. 2001; 1(5):310-23. [Full Text | DOI]
9. Gorgi Z, SheikhFathollahi M, Askarizadeh MK, Rezaeian M. Epidemiology of suicide and attempted suicide in the Larestan and Gerash during 2008 to 2012. J Rafsanjan Univ Med Sci. 2014; 13(7):597-608.

10. Filippatos G, Karasi E. Characteristics of attempted suicide patients presenting to a greek emergency department. Health Sci J. 2017; 11(3):1. [Full Text | DOI]

11. Maselko J, Patel V. Why women attempt suicide: the role of mental illness and social disadvantage in a community cohort study in India. J Epidemiol Community Health. 2008; 62(9):817-22. [Full Text | DOI]

12. Woo JM, Okusaga O, Postolache TT. Seasonality of suicidal behavior. Int J Environ Res Public Health. 2012; 9(2):53147. [Full Text | DOI]

13. Goodwin RD, Marusic A, Hoven CW. Suicide attempts in the United States: the role of physical illness. Soc Sci Med. 2003; 56(8):1783-8. [Full Text | DOI]

14. Scott KM, Hwang I, Chiu WT, Kessler RC, Sampson NA, Angermeyer M, et al. Chronic physical conditions and their association with first onset of suicidal behavior in the world mental health surveys. Psychosom Med. 2010; 72(7):712-9. [Full Text $\mid$ DOI]

15. Pajoumand A, Talaie H, Mahdavinejad A, Birang S, Zarei M, Mehregan FF, et al. Suicide epidemiology and characteristics among young Iranians at poison ward, Loghman-Hakim Hospital (1997-2007). Arch Iran Med. 2012; 15(4). [PubMed | Full Text]

16. Schmidt LE. Age and paracetamol self-poisoning. Gut. 2005; 54(5):686-90. [Full Text | DOI]

\section{AUTHORS CONTRIBUTION}

Following authors have made substantial contributions to the manuscript as under:

ZL: Concept, design, data collection and analysis, manuscript writing and review.

NW: Design, data collection and analysis, manuscript writing and review

K: Design, data collection and analysis, manuscript writing and review

Author agree to be accountable for all respects of the work in ensuring that questions related to the accuracy and integrity of any part of the work are appropriately investigated and resolved.

CONFLICT OF INTEREST

None

GRANT SUPPORT AND FINANCIAL DISCLOSURE

None 